\section{NMR Determination of the Fractal Geometry of Gels Around the Collapse Transition}

R. Blinc, O. Jarh, and A. Zidanšek

J. Stefan Institute, University of Ljubljana, Ljubljana, Yugoslavia

\section{A. Blinc}

University Institute of Gerontology, Internal Clinic Trnovo, Ljubljana, Yugoslavia

\section{Z. Naturforsch. 44a, 163-164 (1989); received September 22, 1988}

The increase in the surface to volume ratio at the collapse transition of gels can be exploited to allow for an NMR determination of the fractal dimension of the gel surface in the liquid state where this quantity is hard to measure with other techniques. The measured quantities are the ratio between the spin-lattice and spin-spin relaxation times $T_{1} / T_{2}$ of the liquid component and the ratio of the masses or volumes of the gel before and after the collapse transition. The technique has been used to determine the surface fractal dimension of a blood plasma gel.

Gels are a form of matter intermediate between solids and liquids. They consist of a tangled polymer network immersed in a liquid, e.g. water $[1,2]$. The water prevents the polymer network from collapsing and the network prevents the water from flowing away. The pore size distribution of the gel is scale invariant and self-similar over two to three orders of magnitude [2].

At high enough temperatures we have a sol consisting of a mixture of monomers and solvent molecules. As the temperature is lowered, partial polymerization takes place. At the sol-gel transition an infinite polymer - i.e. a fractal percolation cluster [3] - appears but there is still a non-negligible amount of small n-mers. At still lower temperatures in the gel state an infinite cross-linked network is formed. Still another possibility is to induce gelation by enzymatic action on monomers or by other external agents. Drastic changes in the state of the gel such as a change in volume - analogous to a phase separation in binary mixtures or a spinodal decomposition - can be produced by small changes in external conditions such as temperature, electric field, $\mathrm{pH}$ or detaching the gel from the test tube surface. The swelling or shrinking of the gel may be continuous or discontinuous $[4,5]$ so that we can speak of a collapse transition.

Here we wish to report how the change in scale and the increase in the surface to volume ratio at the collapse transition can be exploited to allow for an NMR determination of the fractal dimension of the gel surface $D_{\mathrm{s}}$. The observed quantities are the ratio between the spin-lattice $\left(T_{1}\right)$ and spinspin $\left(T_{2}\right)$ relaxation times $T_{1} / T_{2}$ as well as the mass or volume of the gel before and after the collapse.

Reprint requests to Prof. R. Blinc, Jozef Stefan Institute, University of Ljubljana, 61111 Ljubljana/Jugoslawien.
Proton NMR studies $[5,6]$ have established the existence of fast exchange between the water bound to the surface of the gel and the free water. Since the life-time of a water molecule in a given phase is short compared to the spin relaxation time $T_{i k}(i=1,2)$ associated with this phase $(k)$ we have a unique $T_{i}$ characterizing water protons in different environments. We may thus write

$$
\frac{1}{T_{i}}=\left(\frac{1}{T_{i \mathrm{~b}}}\right) \eta+\left(\frac{1}{T_{i \mathrm{f}}}\right)(1-\eta), \quad i=1,2 .
$$

Here $T_{i \mathrm{~b}}$ is the relaxation time of the bound and $T_{i \mathrm{f}}$ the relaxation time of the free water phase, whereas

$$
\eta=\frac{N_{\mathrm{b}}}{N_{\mathrm{b}}+N_{\mathrm{f}}}=K S / V
$$

is the bound water fraction. $N_{\mathrm{b}}$ is the number of water molecules bound to the gel surface, $N_{\mathrm{f}}$ the number of "free" water molecules, $K$ the thickness of the adsorbed water layer, $S$ the surface of the gel and $V$ the total volume of the water.

For free water $T_{1 \mathrm{f}} \approx T_{2 \mathrm{f}}$ whereas this is not the case for bound water, where $T_{2 \mathrm{~b}} \ll T_{1 \mathrm{~b}}$. We thus find from (1)

$$
\frac{T_{1}}{T_{2}}=\frac{1+\left(T_{2 \mathrm{f}} / T_{2 \mathrm{~b}}\right) \eta}{1+\left(T_{1 \mathrm{f}} / T_{1 \mathrm{~b}}\right) \eta} \approx 1+\left(T_{2 \mathrm{f}} / T_{2 \mathrm{~b}}\right) \eta
$$

for $\eta \ll 1$ and $T_{1 \mathrm{f}} / T_{1 \mathrm{~b}} \ll T_{2 \mathrm{f}} / T_{2 \mathrm{~b}}$. Expression (3) allows for a determination of $\eta$ if $T_{2 \mathrm{~b}}$ and $T_{1 \mathrm{~b}}$ are known. If this is not the case we can use the following approximate procedure which is based on the assumption of the validity of the BPP formula [7].

Replacing $1-\eta$ by 1 for $\eta \ll 1$ in (1) we obtain in the BPP approximation

$\Delta\left(\frac{1}{T_{1}}\right)=\frac{1}{T_{1}}-\frac{1}{T_{1 \mathrm{f}}}=\eta \frac{2}{3} K\left[\frac{\tau}{1+\omega_{\mathrm{L}}^{2} \tau^{2}}+\frac{4 \tau}{1+4 \omega_{\mathrm{L}}^{2} \tau^{2}}\right]$

and

$\Delta\left(\frac{1}{T_{2}}\right)=\frac{1}{T_{2}}-\frac{1}{T_{2 \mathrm{f}}}=\eta K\left[\tau+\frac{5 \tau}{3\left(1+\omega_{\mathrm{L}}^{2} \tau^{2}\right)}+\frac{2 \tau}{3\left(1+4 \omega_{\mathrm{L}}^{2} \tau^{2}\right)}\right]$.

Here $\omega_{\mathrm{L}}$ is the proton Larmor frequency whereas $K$ describes the mean squared dipolar interactions within a rotating water molecule. Dividing ( $4 \mathrm{a}$ ) by ( $4 \mathrm{~b})$, we get an equation for $\tau$. Inserting the value of $\tau$ obtained from this equation into (4a) and (4b) we get the bound water fraction $\eta$. Using (1) we can then also obtain $T_{1 \mathrm{~b}}$ and $T_{2 \mathrm{~b}}$.

For a fractal system with a fractal surface dimension $D_{\mathrm{s}}$ and a fractal volume dimension $D_{\mathrm{v}}$,

$$
\eta \propto R^{D_{\mathrm{s}}-D_{\mathrm{v}}}
$$

whereas the mass-volume relation is

$$
m \propto R^{D_{\mathrm{v}}} .
$$

At the collapse or shrinking transition $m$ and $R$ decrease, resulting in an increase in the $T_{1} / T_{2}$ ratio and in $\eta$. We get

$$
\frac{D_{\mathrm{v}}-D_{\mathrm{s}}}{D_{\mathrm{v}}}=\ln \frac{\eta_{2}}{\eta_{1}} / \ln \frac{m_{1}}{m_{2}}
$$

0932-0784/89/0200-0163\$01.30/0. - Please order a reprint rather than making your own copy. 
Table 1. NMR parameters used to determine the surface fractal dimension of blood plasma gel.

\begin{tabular}{|c|c|c|c|c|c|c|c|c|c|}
\hline \multicolumn{5}{|c|}{ Plasma clot (gel) } & \multicolumn{5}{|c|}{ Shrunken plasma clot 2 days after collapse } \\
\hline $\begin{array}{l}\text { Sample } \\
\text { number }\end{array}$ & $m_{1}[\mathrm{~g}]$ & $T_{1}[\mathrm{~ms}]$ & $T_{2}[\mathrm{~ms}]$ & $\eta_{1}$ & $m_{2}[\mathrm{~g}]$ & $T_{1}[\mathrm{~ms}]$ & $T_{2}[\mathrm{~ms}]$ & $\eta_{2}$ & $D_{\mathrm{s}}$ \\
\hline 1 & 0.7076 & 2200 & 520 & $7.2 \times 10^{-3}$ & 0.0487 & 1700 & 160 & $2.2 \times 10^{-2}$ & 1.75 \\
\hline 2 & 0.5636 & 2200 & 520 & 7.2 & 0.0376 & 1800 & 170 & 2.0 & 1.89 \\
\hline 3 & 0.6126 & 2200 & 520 & 7.2 & 0.0308 & 1800 & 160 & 2.0 & 1.95 \\
\hline 4 & 0.6599 & 2100 & 520 & 7.8 & 0.0570 & 1700 & 180 & 2.0 & 1.82 \\
\hline average & & & & $7.3 \times 10^{-3}$ & & & & $2.0 \times 10^{-2}$ & 1.86 \\
\hline
\end{tabular}

Expression (6) allows for a simple and direct determination of the difference between the volume and surface fractal dimensions, which is hard to measure with other techniques in the liquid state. It should be noticed that the fractal dimension is independent of the dipolar interaction constant $K$ as it depends only on the ratio of $\eta_{2} / \eta_{1}$ and not on $\eta_{1}$ or $\eta_{2}$ separately.

The system studied was human blood plasma anticoagulated with $\mathrm{Na}_{2}$-EDTA $3 \mathrm{mmol} / 1$, thrombin induced gels of the above systems and retracted plasma clots. The retraction - or collapse - was induced by separating the gel from the sample tube wall. In the above gel the fibrin polymer network constitutes only about $0.3 \%$ of the volume of the gel.

Since the polymer constitutes only a very small fraction of the volume of the gel, one may safely assume that the masslength scale relation for the water can be approximated by $D_{\mathrm{v}}=3$. The properties of the "bound" water fraction are however determined by the gel surface so that (6) can be used for a determination of the surface fractal dimension of the gel in the liquid state.

[1] P. J. Flory, Disc. Faraday Soc. 57, 7 (1974).

[2] T. Tanaka, G. Swislow, and I. Ohmine, Phys. Rev. Lett 42, 1556 (1979). For a determination of the fractal geometry of dried aerogels see E. Courtens and R. Vacher, Z. Phys. B - Condensed Matter 68, 355 (1987).

[3] R. Orbach, J. Stat. Phys. 36, 735 (1984).

[4] B. Derrida and H. J. Herrmann, J. Physique 44, 1365 (1983).
The measurements were performed at a proton Larmor frequency of $v_{\mathrm{L}}=86.55 \mathrm{MHz}$. The spin-lattice relaxation times $T_{1}$ were measured by the $180^{\circ}-\tau-90^{\circ}$ and the spin-spin relaxation times $T_{2}$ by the Carr-Purcell sequence with a $40 \mathrm{~ms}$ spacing between the 180 degree pulses. The decay of the transverse and the recovery of the longitudinal magnetizations were found to be monoexponential. The results for four different blood plasma samples are collected in Table 1. The "free water" $T_{1}$ and $T_{2}$ were determined as $T_{1 \mathrm{f}}=2900 \mathrm{~ms}$ and $T_{2 \mathrm{f}}=2000 \mathrm{~ms}$. The average bound water fraction was found to be $\eta_{1}=7.3 \times 10^{-3}$ for the plasma gel and $\eta_{2}=$ $2.0 \times 10^{-2}$ for the retracted plasma gel.

The surface fractal dimensions for the four samples were found to vary between $D_{\mathrm{s}}=1.75$ and $D_{\mathrm{s}}=1.95$. The average over the four different samples yields $D_{\mathrm{s}}=1.86$. This may be compared with the value of $D_{\mathrm{s}}$ for a Sierpinski carpet where $D_{\mathrm{s}}=\ln 8 / \ln 3=1.89$. The internal surface of the plasma gel is thus full of pores and rather open.

[5] F. Tabak, M. Corti, L. Pavesi, and A. Rigamonti. J. Phys. C 20, 5691 (1987).

[6] J. R. Zimmermann and W. E. Brittin, J. Phys. Chem. 61, 1328 (1957).

[7] A. Abragam, The Principles of Nuclear Magnetism, Clarendon Press, Oxford 1961. 